\title{
Método de investimento em bolsa de valores de empresas do agronegócio via diferenciação discreta
}

\author{
Luís Roberto Almeida Gabriel Filho ${ }^{1}$, Camila Pires Cremasco ${ }^{2}$, Paulo Victor de Souza Aoki ${ }^{3}$, Thiago Ceber \\ de $\mathrm{Mello}^{4}$, Leandro Pereira de Moraes ${ }^{4}$ \\ ${ }^{1}$ Docente e Assessor Científico da UNOESTE, Presidente Prudente, SP; ${ }^{2}$ Docente do Departamento de Matemática, \\ FATEC, Presidente Prudente, SP; ${ }^{3}$ Docente da ETEC de Adamantina, SP; ${ }^{4}$ Bacharel em Ciência da Computação, FAI, \\ Adamantina.
}

\section{Resumo}

Um dos grandes desejos de investidores da Bolsa de Valores é prever o comportamento das ações de empresas visando maior lucro em suas aplicações. A análise realizada neste trabalho estabelece um método de investimento de maneira que os acionistas possam seguir visando lucro de investimento em empresas, através de teoremas de caráter inéditos, tais como os testes da derivada primeira e segunda para sistemas discretos, uma vez que os registros dos preços das bolsas são pontos discretos em intervalos de 15 minutos. Sendo assim, o objetivo deste estudo é o estabelecimento de métodos sobre derivadas discretas, visando definir e desenvolver teoremas e testes para estimava da localização de máximos e mínimos de valores de ações de empresas. Foram utilizadas oscilações na bolsa de valores de três empresas brasileiras pertencentes ao agronegócio sul-americano, que as denominaremos por $\mathrm{A}, \mathrm{B}$ e $\mathrm{C}$, com participação em derivados de carnes, massas e cana de açúcar. Em geral, considerou-se a função $f: \mathrm{N} . h \rightarrow R$ onde $h>0$ é um número real fixo e $\mathrm{N} h=\{n . h: n \in \mathrm{N}\}$, definindo a "derivada discreta" de $f$ no ponto $n h$ por: $\dot{f}(n h)=\frac{f((n+1) \cdot h)-f(n h)}{h}$. Nas simulações reais efetuadas, se o investidor procedesse a suas transações comerciais de acordo com o método seria obtido um lucro de $1,2 \%$ em ações da empresa $A, 1,4 \%$ na empresa $B$ e $0,7 \%$ na empresa $C$ nos datas escolhidas para a análise. Como o lucro obtido diário nas simulações foi maior em torno de $1 \%$, concluímos que o método é extremamente eficiente, visto que este é o rendimento aproximado mensal de fundos em geral existentes no mercado financeiro.

Palavras-Chave: sistemas discretos, localização de máximos e mínimos, mercado financeiro.

\section{Method of investment in stock exchange of agribusiness companies saw differentiation discrete}

\begin{abstract}
One of the great desires of investors of the Stock Exchange is predict the behavior of the shares of companies seeking higher profits in its applications. One of the great desires of investors in stock exchange is predict the behavior of the shares of companies seeking higher profits in its applications. The analysis performed here provides a method of investment so that shareholders can follow to profit from investment in business by theorems that we believe are unprecedented, such as tests of the first and second derivative for discrete systems, since the records of prices of the bags are discrete points at intervals of 15 minutes. Therefore, the objective of this study is the establishment of methods of deriving discrete, to define and develop theorems and tests to estimate the location of maximum and minimum values of shares of companies. Oscillations were used in the stock exchange in three Brazilian companies belonging to the South American agribusiness, which called A, B and C, with participation in derivatives of meat, pasta and sugar cane. In general, it is the function $f: \mathrm{N} . h \rightarrow R$ which $h>0$ is a fixed real number and $\mathrm{N} h=\{n . h: n \in \mathrm{N}\}$ define the "discrete derivative" from $f$ the point $n h$ of: $\dot{f}(n h)=\frac{f((n+1) \cdot h)-f(n h)}{h}$. In actual simulations performed, if the investor to carry out their business transactions in accordance with the method could be obtained a profit of $1.2 \%$ in shares of company $A$, company $B$ in $1.4 \%$ and $0.7 \%$ in the company $\mathrm{C}$ in dates chosen for analysis. As the simulations in daily profit was higher at around $1 \%$, we find that the method is extremely efficient, since this is the approximate monthly income of the general funds in the financial market.
\end{abstract}

Keywords: discrete systems, localization of maximums and minimums, financial market. 


\section{Introdução}

Um dos grandes desejos de investidores da Bolsa de Valores é prever o comportamento das ações de empresas visando maior lucro em suas aplicações.

O cálculo diferencial integral fornece condições por meio de conceitos de derivadas para estudo de taxa de variação de funções contínuas (LEITHOLD, 1994). É uma teoria extremamente útil para a detecção de pontos de máximo e mínimo relativos. Estudos nas áreas de sistemas discretos estão revelando-se extremamente eficazes nos campos da computação, onde são desenvolvidos sistemas muitas vezes mais eficientes que sistemas contínuos.

A análise aqui feita estabelece um método de maneira que os acionistas possam seguir visando lucro de investimento em empresas do agronegócio. Também foram desenvolvidos teoremas inéditos, tais como os testes da derivada primeira e segunda para sistemas discretos.

Observa-se que as variáveis envolvidas na variação de cotas em bolsa de valores são inúmeras. Desta forma, o método desenvolvido neste trabalho tem um maior êxito se aplicado em ações estáveis.

Registros dos preços das bolsas são pontos discretos em intervalos de 15 minutos, sendo preponderantemente necessária a utilização de teoremas na forma discretas e não na forma continuas.

O objetivo deste estudo é o estabelecimento de métodos sobre derivadas discretas, visando definir e desenvolver teoremas e testes para estimava da localização de máximos e mínimos de valores de ações de empresas do agronegócio. Estes teoremas desenvolvidos foram aplicados em bolsa de valores, no sentido de mostrar as melhores ocasiões de compra e venda de ações. Foram utilizadas oscilações na bolsa de valores de três empresas brasileiras pertencentes ao agronegócio sul-americano, que são denotadas por A, B e C, com participação em derivados de carnes, massas e cana de açúcar.

O agronegócio ou complexo agroindustrial possui três grandes setores: (1. $\stackrel{\circ}{)})$ negócios agropecuários propriamente ditos (produtores rurais); (2.ํ) negócios descritos pelas indústrias e comércios que fornecem insumos para os negócios agropecuários (fabricantes de fertilizantes e defensivos químicos); (3.ํ) negócios que compram os produtos agropecuários, os beneficiam, os transportam e os vendem para os consumidores finais (frigoríficos, as fábricas de fiação, curtumes e supermercados, etc).

Sendo assim, o conceito de agronegócio representa o enfoque moderno que considera todas as empresas que produzem, processam, e distribuem produtos agropecuários.

Tais empresas possuem, em geral, ações na bolsa de valores, que figura objeto deste estudo que, segundo Medeiros (1987), são os mais importantes centros de negociação de ações, devido ao expressivo volume e maior transparência das operações organizadas como associações civis, sem fins lucrativos e com funções de interesse público.

\section{Material e métodos}

Neste trabalho foram estabelecidos teoremas do cálculo diferencial integral para a detecção de extremos relativos em sistemas discretos, os quais foram aplicados em bolsa de valores, no sentido de mostrar as melhores ocasiões de compra e venda de ações. Foram utilizadas oscilações na bolsa de valores de três empresas brasileiras pertencentes ao agronegócio sul-americano, denotadas por A, B e C.

A empresa A tem participação expressiva nos segmentos de industrializados e congelados 
de carne, tais como: lingüiça, salsicha, mortadela, hambúrguer, almôndegas, quibes e cortes, possuindo uma receita líquida de $R \$ 4,9$ bilhões.

$A$ empresa $B$ atua também no segmento agroindustrial na produção de alimentos derivados de carnes suína, bovina, de frango e de peru, além de massas e margarinas. É uma das maiores empresas de produção e exportação de alimentos da América Latina.

A empresa $C$ é o maior grupo individual do mundo no que se refere à produção de derivados da cana-de-açúcar, com capacidade para moer mais de 40 milhões de toneladas de cana e responsável por $12 \%$ da produção total da região centro-sul do Brasil.

Os dados analisados foram obtidos por meio de software Home-Broker on-line da BOVESPA sobre a Bolsa de valores que, diariamente, informa o preço das ações de varias empresas do ramo pertencente ao agronegócio brasileiro.

Para cada empresa estudada foram estabelecidas determinadas datas que foram denominadas "dias com oscilação suave". Desta maneira a grande eficácia do método proposto é sua aplicação em períodos com certa estabilidade econômica. A data para as empresas A, B e C são 06 e 13/10/2006.

\section{Resultados e discussão}

Inicialmente, serão reestruturados teoremas e definições do Cálculo Diferencial Integral para sistemas contínuos, definindo e apresentando teoremas para funções discretas e para a determinação da localização de máximos e mínimos de tais funções.

A etapa anterior se faz necessária já que o estudo em questão se trata de uma aplicação na bolsa de valores em funções que variam de acordo com o tempo. Vale ressaltar que os resultados para sistemas discretos estabelecidos são voltados a ao estabelecer um método de venda e compra de ações, que naturalmente, devem ser feitos em pontos de mínimo e máximo, respectivamente, em relação aos preços das mesmas.

\section{Resultados teóricos}

Inicialmente, considera-se a função $f:$ N. $h \rightarrow R$ onde $h>0$ é um número real fixo e $\mathrm{N} h=\{n . h: n \in \mathrm{N}\}$. Segundo Gabriel Filho (2004), pode-se definir a "derivada discreta" de $f$ no ponto $n h, n \in \mathrm{N}$, denotada por $\dot{f}$, da seguinte forma:

$$
\dot{f}(n h)=\frac{f((n+1) \cdot h)-f(n h)}{h} .
$$

Desta forma, é possível definir a derivada de ordem 2 de funções discretas utilizando o mesmo processo. Defini-se a "derivada segunda discreta" de $f$ no ponto $n h, n \in \mathrm{N}$, denotada por $\ddot{f}$, da forma:

$$
\ddot{f}(n h)=\frac{\dot{f}((n+1) \cdot h)-\dot{f}(n h)}{h} .
$$

Assim, é possível escrever:

$$
\ddot{f}(n h)=\frac{f((n+2) h)-2 f((n+1) h)+f(n h)}{h^{2}} .
$$

Em funções contínuas, pontos críticos são essencias na determinação de máximos e mínimos e são definidos fundamentalmente onde a derivada é nula ou não existe. Em sistemas discretos, neste trabalho, será estabelecida a definição a seguir. 
Definição: Seja $N$ o conjunto de todos os números naturais e seja $\mathrm{A} \subset \mathrm{N} h, h>0$, tal que: $\mathrm{A}=\left\{n_{0} h,\left(n_{0}+1\right) h, \ldots,\left(n_{1}-1\right) h, n_{1} h\right\}$, com $n_{0}, n_{1} \in \mathrm{N}$. Seja $p \in \mathrm{A}$ tal que $p=\bar{n} h$. Diz-se que $p$ é um "ponto crítico discreto" para a função $f$ se $\bar{n}=n_{0}$, ou $\bar{n}=n_{1}$, ou $\dot{f}((\bar{n}-1) h)<0 \quad$ e $\quad \dot{f}(p)>0 \quad$, ou $\dot{f}((\bar{n}-1) h)>0 \quad$ e $\quad \dot{f}(p)<0, \quad$ ou $\dot{f}((\bar{n}-1) h)=0 \quad$ e $\quad \dot{f}(p)>0 \quad$ ou $\dot{f}((\bar{n}-1) h)=0$ e $\dot{f}(p)<0$.

Os resultados clássicos de determinação de máximos e mínimos são relacionados à sua localização em função de tais pontos críticos. Para isto, devem-se definir máximos e mínimos em funções discretas.

Definição: Seja $\mathrm{A} \subset \mathrm{N} h, h>0$ tal que: $\mathrm{A}=\{$ $\left.n_{0} h, \quad\left(n_{0}+1\right) h, \ldots \quad, \quad\left(n_{1}-1\right) h, \quad n_{1} h \quad\right\}, \quad$ com $n_{0}, n_{1} \in \mathrm{N}$. Seja $p \in \mathrm{A}$ tal que $p=\bar{n} h$ e $\bar{n} \neq n_{0}, \bar{n} \neq n_{1}$. A função $f$ terá um:

(i) valor mínimo relativo em $p$ se:

$$
\begin{aligned}
& f(p)<f((\bar{n}-1) h) \\
& f(p)<f((\bar{n}+1) h) ;
\end{aligned}
$$

(ii) valor máximo relativo em $p$ se:

$$
\begin{aligned}
& f(p)>f((\bar{n}-1) h) \\
& f(p)>f((\bar{n}+1) h) .
\end{aligned}
$$

Se a função $f$ possuir um valor de máximo relativo ou mínimo relativo em $p$, então diz-se que $f$ possui um valor de extremo relativo em $p$.

Desta forma, é possível estabelecer resultados voltados à localização dos pontos de máximos e mínimos em funções discretas. Os teoremas enunciados a seguir constituem a base do método que será proposto para compra e venda de ações, uma vez que buscam determinar os extremos relativos de funções discretas, um dos objetivos do presente estudo.

Teorema 1. (Teste da derivada primeira discreta para extremos relativos): Seja $\mathrm{A} \subset \mathrm{N} h, h>0$ tal que: $\mathrm{A}=\left\{n_{0} h,\left(n_{0}+1\right) h, \ldots,\left(n_{1}-1\right) h, n_{1} h\right.$ \}, com $n_{0}, n_{1} \in \mathrm{N}$. Seja $p \in \mathrm{A}$ tal que $p=\bar{n} h$ e $\bar{n} \neq n_{0}, \bar{n} \neq n_{1}$. Então, se:

(i) $\dot{f}((\bar{n}-1) h)<0$ e $\dot{f}(p)>0$, então $f$ terá um valor mínimo relativo em $p$.

(ii) $\dot{f}((\bar{n}-1) h)>0$ e $\dot{f}(p)<0$, então $f$ terá um valor máximo relativo em $p$.

Seja $\mathrm{A} \subset \mathrm{N} h, \quad h>0$ tal que: $\mathrm{A}=\left\{n_{0} h\right.$, $\left.\left(n_{0}+1\right) h, \ldots,\left(n_{1}-1\right) h, n_{1} h\right\}$, com $n_{0}, n_{1} \in \mathrm{N}$. Seja $p \in \mathrm{A}$ tal que $p=\bar{n} h$. Se $p$ é um ponto crítico, então $\bar{n}=n_{0}$, ou $\bar{n}=n_{1}$, ou $p$ é um extremo relativo.

Teorema 2. (Teste da derivada segunda discreta para extremos relativos):: Seja $\mathrm{A} \subset \mathrm{N} h, h>0$ tal que: $\mathrm{A}=\left\{n_{0} h,\left(n_{0}+1\right) h, \ldots,\left(n_{1}-1\right) h, n_{1} h\right.$ \}, com $n_{0}, n_{1} \in \mathrm{N}$. Seja $p \in \mathrm{A}$ um ponto crítico tal que $p=\bar{n} h$ e $\bar{n} \neq n_{0}, \bar{n} \neq n_{1 .}$. Assim,

(i) se $\ddot{f}((\bar{n}-1) h)>0$, então $f$ tem um valor mínimo relativo em $p$.

(ii) se $\ddot{f}((\bar{n}-1) h)<0$, então $f$ tem um valor máximo relativo em $p$.

No modelo apresentado neste trabalho, não foram realizadas duas compras seguidas e também duas vendas seguidas. Observa-se que caso as ações forem compradas e não haver oportunidades segundo as considerações (1) e 
(2) para que ocorra a venda, e também se o pregão fechar com um valor acima do valor referido comprado, então serão vendidas ações. Também se considera que não serão compradas ações no ponto $p=n_{0} h$, para $\mathrm{A} \subset \mathrm{N} h, h>0$ tal que: $\mathrm{A}=\left\{n_{0} h,\left(n_{0}+1\right) h, \ldots,\left(n_{1}-1\right) h, n_{1} h\right.$ \} , com $n_{0}, n_{1} \in \mathrm{N}$. Seja $p \in \mathrm{A}$ tal que $p=\bar{n} h$ e $\bar{n} \neq n_{0}, \bar{n} \neq n_{1}$. Então, se:

(i) $\quad p$ for um ponto de mínimo relativo, então é conveniente a compra de ações em $(\bar{n}+1) h$.

(ii) $\quad p$ for um ponto de máximo relativo então é conveniente a venda de ações em $(\bar{n}+1) h$.

As propostas apresentadas se tratam de mecanismos eficientes de gestão financeira para aplicação em bolsa de valores. Tais propostas foram implementadas de forma prática nas empresas A, B e C em estudo. Além, disto, ao longo da apresentação dos resultados práticos nas empresas a seguir, será estabelecida uma relação que retorna o lucro líquido (quando o caso) da movimentação financeira no período considerado.

\section{Simulações em empresa do setor agroindustrial}

Nesta parte do estudo, foi realizada a aplicação dos métodos desenvolvidos nas ações da empresa A no dia 06/10/2006. Assim, considerou-se $n h$ como sendo uma variável temporal, representando os minutos a partir de 10:00 horas. Também considerou-se que a imagem dos pontos $\{n h \in \mathrm{N} h: 0 \leq n \leq 28\}$, ou seja, os valores de $f(n h)$ como valores em reais $(\mathrm{R} \$)$ de ações.

Tabela 1. Valores das ações da empresa A no dia 06/10/2006 e aplicação dos métodos desenvolvidos no presente estudo.

\begin{tabular}{|c|c|c|c|c|c|c|c|}
\hline$n$ & Tempo (nh) & $f(n h)$ & $f^{\prime} .10^{-3}$ & $\begin{array}{l}\text { Pontos } \\
\text { Críticos }\end{array}$ & $\ddot{f} .10^{-3}$ & Extremo Relativo & Compra / Venda \\
\hline 0 & $10: 00$ & 24,56 & 0,0 & $\mathrm{x}$ & $-0,8$ & $\operatorname{Max}$ & \\
\hline 1 & $10: 15$ & 24,56 & $-12,7$ & & $-0,4$ & & \\
\hline 2 & $10: 30$ & 24,37 & $-19,3$ & & 1,8 & & \\
\hline 3 & $10: 45$ & 24,08 & 8,0 & $x$ & $-0,2$ & Min & \\
\hline 4 & 11:00 & 24,2 & 5,3 & & $-1,2$ & & C \\
\hline 5 & $11: 15$ & 24,28 & $-12,0$ & $x$ & 1,4 & $\operatorname{Max}$ & \\
\hline 6 & $11: 30$ & 24,1 & 9,3 & $\mathrm{x}$ & $-0,6$ & Min & V \\
\hline 7 & $11: 45$ & 24,24 & 0,7 & & $-0,7$ & & $\mathrm{C}$ \\
\hline 8 & $12: 00$ & 24,25 & $-10,0$ & $x$ & 0,5 & $\operatorname{Max}$ & \\
\hline 9 & $12: 15$ & 24,1 & $-2,0$ & & $-0,1$ & & $\mathrm{~V}$ \\
\hline 10 & $12: 30$ & 24,07 & $-4,0$ & & $-0,1$ & & \\
\hline 11 & $12: 45$ & 24,01 & $-5,3$ & & 0,7 & & \\
\hline 12 & 13:00 & 23,93 & 4,7 & $x$ & $-0,6$ & Min & \\
\hline 13 & $13: 15$ & 24 & $-4,7$ & $x$ & 0,6 & $\operatorname{Max}$ & $C$ \\
\hline 14 & 13:30 & 23,93 & 4,0 & $x$ & $-0,3$ & Min & $\mathrm{V}$ \\
\hline 15 & $13: 45$ & 23,99 & 0,0 & $x$ & 0,2 & Sela & $\mathrm{C}$ \\
\hline 16 & $14: 00$ & 23,99 & 3,3 & & $-0,5$ & & $\mathrm{~V}$ \\
\hline 17 & $14: 15$ & 24,04 & $-4,7$ & $x$ & 0,4 & $\operatorname{Max}$ & \\
\hline 18 & $14: 30$ & 23,97 & 2,0 & $x$ & $-0,3$ & Min & \\
\hline 19 & $14: 45$ & 24 & $-2,7$ & $x$ & 0,8 & $\operatorname{Max}$ & $C$ \\
\hline 20 & 15:00 & 23,96 & 9,3 & $x$ & $-0,2$ & Min & $\mathrm{V}$ \\
\hline 21 & $15: 15$ & 24,1 & 6,7 & & 0,2 & & $\mathrm{C}$ \\
\hline
\end{tabular}




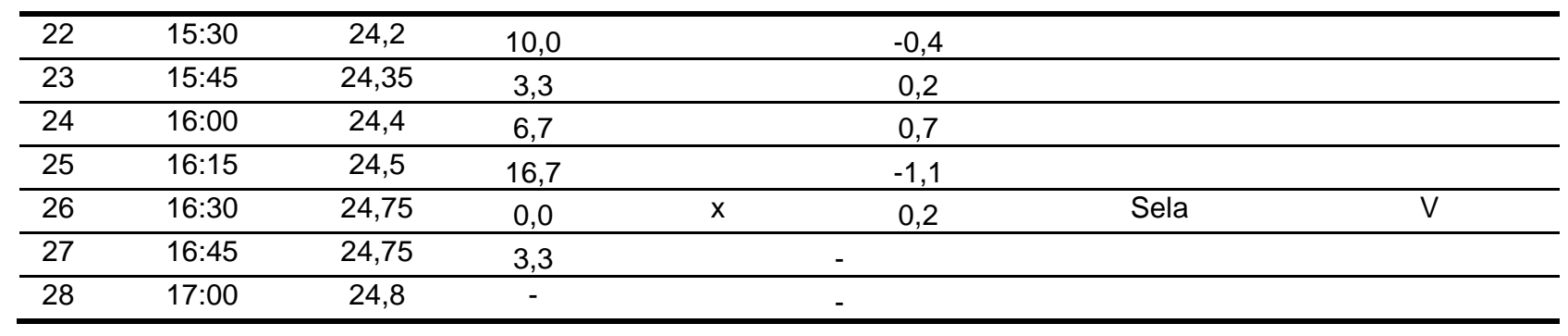

Observa-se que, para encontrar os pontos críticos descritos na Tabela 1, é necessário utilizar o Teorema 1 sobre resultados a cerca de derivadas discretas. Além disto, vale ressaltar que se é investido um montante de $M$ reais em ações, cujo preço unitário de cada ação fosse $r$ reais, então compra-se $\left(\frac{M}{r}\right)$ ações. A Figura 1 ilustra o comportamento das ações.

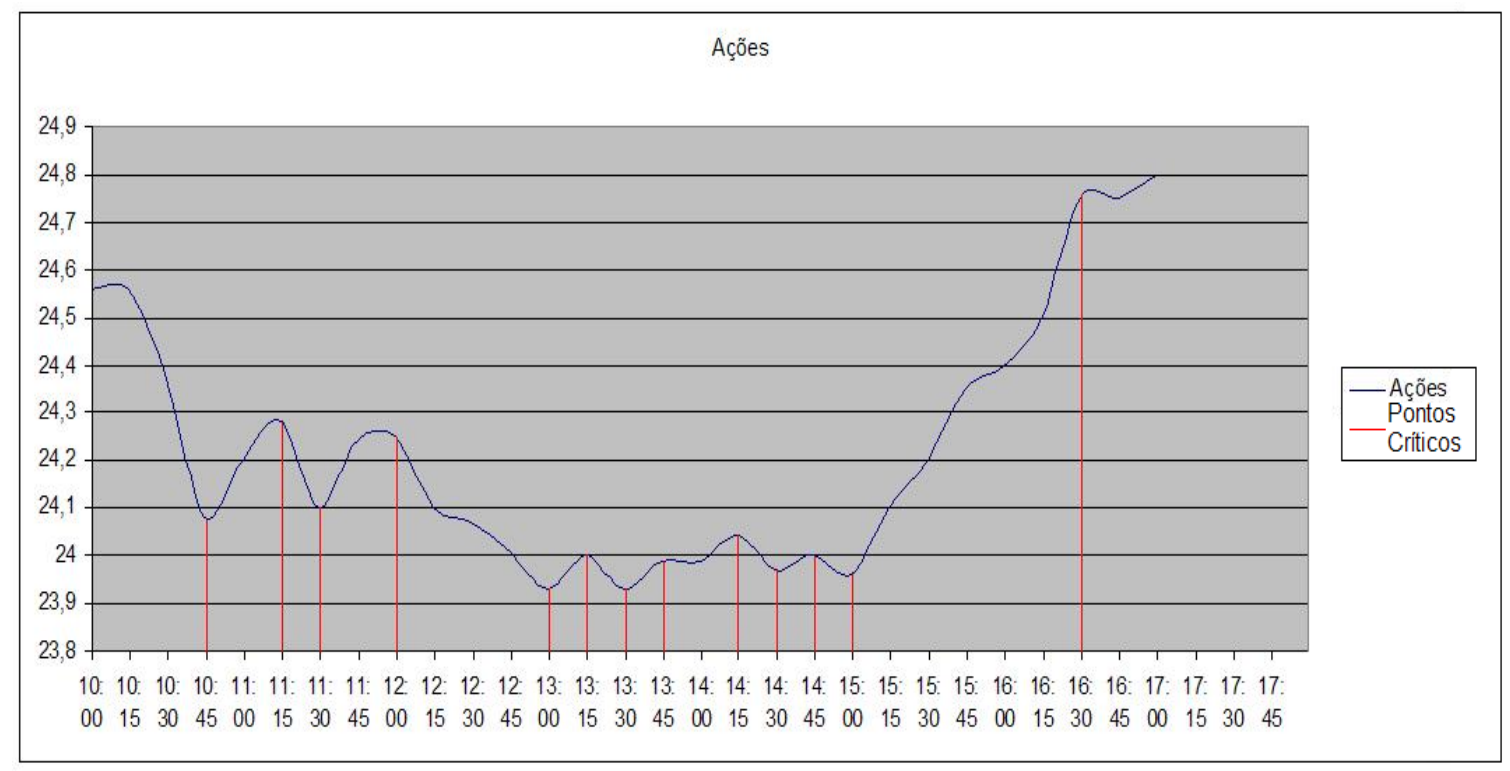

Figura 1. Indicações dos pontos críticos das oscilações nas ações da empresa A.

Será utilizada a seguinte terminologia: "se ocorre a primeira ocasião de compra de ações, o valor neste instante da ação será denotado por $C_{1}$; na segunda ocasião, o valor será por $C_{2}$, e assim por diante".

Visto que neste método é necessário comprar ações antes de vender, será denotado por $V_{1}$ o valor da ação na primeira ocasião de oportunidade de venda; por $V_{2}$ o valor da ação na segunda ocasião, e assim por diante.
Logo, se $M$ é o montante investido na bolsa, tem-se que, no final do "pregão", houve um retorno de:

$$
\frac{V_{1} V_{2} V_{3} V_{4} V_{5} V_{6}}{C_{1} C_{2} C_{3} C_{4} C_{5} C_{6}} M=
$$

$$
\begin{gathered}
\frac{(24,10) \cdot(24,10) \cdot(23,93) \cdot(23,99) \cdot(23,96)(24,75)}{(24,20) \cdot(24,24) \cdot(24,00) \cdot(23,99) \cdot(24,00) \cdot(24,10)} M \\
\cong 1,0122 \cdot M,
\end{gathered}
$$

ou seja, um lucro líquido de 1,22\%. 
Da mesma forma será realizada a aplicação dos métodos desenvolvidos nas ações da empresa B no dia 13/10/2006, conforme a Tabela 2.

Tabela 2. Valores das ações da empresa B no dia 13/10/2006 e aplicação dos métodos desenvolvidos no presente estudo.

\begin{tabular}{|c|c|c|c|c|c|c|c|}
\hline$n$ & $\begin{array}{c}\text { Tempo } \\
(n h)\end{array}$ & $f(n h)$ & $\dot{f} .10^{-3}$ & $\begin{array}{l}\text { Pontos } \\
\text { Críticos }\end{array}$ & $\ddot{f} .10^{-3}$ & $\begin{array}{l}\text { Extremo } \\
\text { Relativo }\end{array}$ & $\begin{array}{c}\text { Compra I } \\
\text { Venda }\end{array}$ \\
\hline 0 & $10: 00$ & 6,37 & 1,3 & & 0,0 & & \\
\hline 1 & $10: 15$ & 6,39 & 1,3 & & 0,0 & & \\
\hline 2 & 10:30 & 6,41 & 1,3 & & $-0,2$ & & \\
\hline 3 & $10: 45$ & 6,43 & $-2,0$ & $x$ & 0,2 & Max & \\
\hline 4 & 11:00 & 6,4 & 0,7 & $x$ & 0,0 & Min & \\
\hline 5 & 11:15 & 6,41 & 0,0 & $x$ & 0,1 & Sela & $\mathrm{C}$ \\
\hline 6 & 11:30 & 6,41 & 1,3 & & 0,0 & & \\
\hline 7 & $11: 45$ & 6,43 & 1,3 & & 0,1 & & \\
\hline 8 & $12: 00$ & 6,45 & 2,7 & & $-0,4$ & & \\
\hline 9 & 12:15 & 6,49 & $-2,7$ & $x$ & 0,2 & Max & \\
\hline 10 & 12:30 & 6,45 & 0,7 & $x$ & $-0,1$ & Min & $\mathrm{V}$ \\
\hline 11 & 12:45 & 6,46 & $-1,3$ & $x$ & 0,1 & Max & $\mathrm{C}$ \\
\hline 12 & 13:00 & 6,44 & 0,0 & $x$ & 0,2 & Min & $\mathrm{V}$ \\
\hline 13 & $13: 15$ & 6,44 & 2,7 & & $-0,2$ & & $C$ \\
\hline 14 & 13:30 & 6,48 & 0,0 & $x$ & 0,0 & Sela & $\mathrm{V}$ \\
\hline 15 & $13: 45$ & 6,48 & 0,0 & $x$ & $-0,2$ & Sela & \\
\hline 16 & 14:00 & 6,48 & $-2,7$ & $x$ & 0,3 & Max & \\
\hline 17 & $14: 15$ & 6,44 & 1,3 & $x$ & 0,1 & Min & \\
\hline 18 & $14: 30$ & 6,46 & 2,7 & & $-0,3$ & & $C$ \\
\hline 19 & 14:45 & 6,5 & $-1,3$ & $x$ & 0,1 & $\operatorname{Max}$ & \\
\hline 20 & $15: 00$ & 6,48 & 0,0 & $x$ & 0,0 & Min & $\mathrm{V}$ \\
\hline 21 & $15: 15$ & 6,48 & 0,0 & $x$ & $-0,3$ & Sela & \\
\hline 22 & $15: 30$ & 6,48 & $-4,0$ & & 0,4 & & \\
\hline 23 & $15: 45$ & 6,42 & 2,0 & $x$ & $-0,1$ & Min & \\
\hline 24 & $16: 00$ & 6,45 & 0,7 & & $-0,2$ & & $\mathrm{C}$ \\
\hline 25 & $16: 15$ & 6,46 & $-2,7$ & $x$ & 0,1 & Max & \\
\hline 26 & $16: 30$ & 6,42 & $-1,3$ & & 0,4 & & \\
\hline 27 & $16: 45$ & 6,4 & 4,0 & $x$ & 0,0 & & \\
\hline 28 & 17:00 & 6,46 & - & & 0,0 & & $\mathrm{~V}$ \\
\hline
\end{tabular}

Neste caso, obteve-se um lucro líquido de:

$$
\frac{V_{1} V_{2} V_{3} V_{4} V_{5}}{C_{1} C_{2} C_{3} C_{4} C_{5}} M=1,405 \%
$$

A Figura 2 ilustra o comportamento das ações da empresa B. 


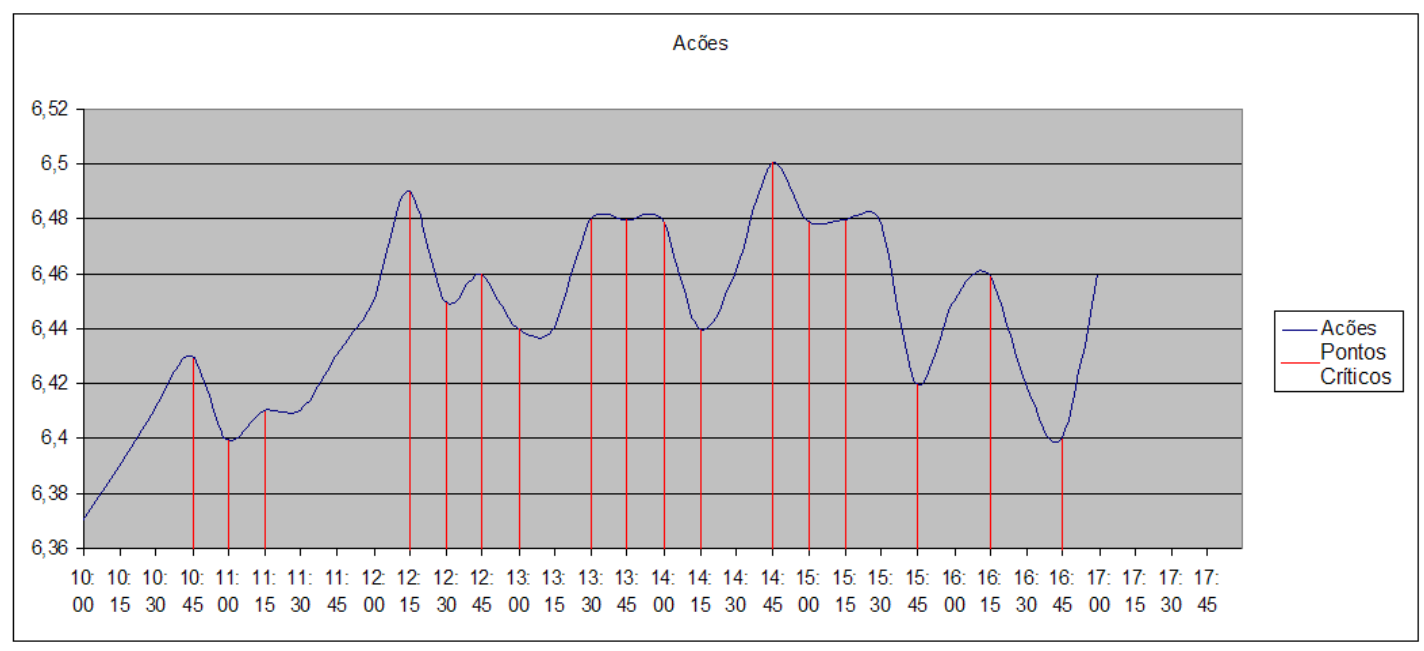

Figura 2. Indicações dos pontos críticos das oscilações nas ações da empresa B.

Por fim será aplicado o método desenvolvido nas ações da empresa $\mathrm{C}$ no dia 13/10/2006, conforme a Tabela 3.

Tabela 3. Valores das ações da empresa C no dia 13/10/2006 e aplicação dos métodos desenvolvidos no presente estudo.

\begin{tabular}{|c|c|c|c|c|c|c|c|}
\hline$n$ & Tempo (nh) & $f(n h)$ & $f^{\prime} \cdot 10^{-3}$ & Pontos Críticos & $\ddot{f} .10^{-3}$ & $\begin{array}{l}\text { Extremo } \\
\text { Relatívo }\end{array}$ & $\begin{array}{c}\text { Compra I } \\
\text { Venda }\end{array}$ \\
\hline 0 & $\begin{array}{l}10: 00 \\
\end{array}$ & 35,7 & $-13,3$ & & 1,3 & & \\
\hline 1 & $10: 15$ & 35,5 & 6,7 & $x$ & $-0,2$ & Min & \\
\hline 2 & $10: 30$ & 35,6 & 3,3 & & 0,4 & & $\mathrm{C}$ \\
\hline 3 & 10:45 & 35,65 & 10,0 & & $-0,2$ & & \\
\hline 4 & $11: 00$ & 35,8 & 6,7 & & 0,1 & & \\
\hline 5 & $11: 15$ & 35,9 & 8,7 & & 0,2 & & \\
\hline 6 & $11: 30$ & 36,03 & 11,3 & & $-0,3$ & & \\
\hline 7 & 11:45 & 36,2 & 6,7 & & $-0,8$ & & \\
\hline 8 & $12: 00$ & 36,3 & $-5,3$ & $x$ & 0,7 & Max & \\
\hline 9 & $12: 15$ & 36,22 & 5,3 & $\mathrm{X}$ & $-0,8$ & Min & $\mathrm{V}$ \\
\hline 10 & $12: 30$ & 36,3 & $-6,7$ & $\mathrm{X}$ & 0,4 & $\operatorname{Max}$ & C \\
\hline 11 & $12: 45$ & 36,2 & 0,0 & $\mathrm{X}$ & 0,0 & Sela & $\mathrm{V}$ \\
\hline 12 & 13:00 & 36,2 & $-0,7$ & & 0,5 & & \\
\hline 13 & $13: 15$ & 36,19 & 7,3 & $X$ & $-0,9$ & Min & \\
\hline 14 & $13: 30$ & 36,3 & $-6,7$ & $\mathrm{X}$ & 0,7 & $\operatorname{Max}$ & $C$ \\
\hline 15 & $13: 45$ & 36,2 & 3,3 & $\mathrm{X}$ & $-0,4$ & Min & $\mathrm{V}$ \\
\hline 16 & 14:00 & 36,25 & $-3,3$ & $X$ & 0,2 & $\operatorname{Max}$ & $\mathrm{C}$ \\
\hline 17 & $14: 15$ & 36,2 & 0,0 & $\mathrm{X}$ & $-0,8$ & Sela & $\mathrm{V}$ \\
\hline 18 & $14: 30$ & 36,2 & $-12,7$ & & 1,7 & & \\
\hline 19 & $14: 45$ & 36,01 & 12,7 & $X$ & $-1,3$ & Min & \\
\hline 20 & 15:00 & 36,2 & $-6,7$ & $X$ & 0,4 & $\operatorname{Max}$ & C \\
\hline 21 & $15: 15$ & 36,1 & 0,0 & $\bar{X}$ & 1,0 & Min & $\mathrm{V}$ \\
\hline 22 & $15: 30$ & 36,1 & 15,3 & & $-1,2$ & & C \\
\hline 23 & 15:45 & 36,33 & $-3,3$ & $X$ & $-0,6$ & Max & \\
\hline 24 & $16: 00$ & 36,28 & $-12,0$ & & 1,2 & & $\mathrm{~V}$ \\
\hline
\end{tabular}




\begin{tabular}{cccccccc}
\hline 25 & $16: 15$ & 36,1 & 6,0 & $X$ & $-1,2$ & Min & \\
\hline 26 & $16: 30$ & 36,19 & $-12,7$ & $X$ & 0,8 & Max & $\mathrm{C}$ \\
\hline 27 & $16: 45$ & 36 & 0,0 & $X$ & - & & $\mathrm{V}$ \\
\hline 28 & $17: 00$ & 36 & - & & - & & \\
\hline
\end{tabular}

Neste caso, obteve-se um lucro líquido de $0,7338 \%$, pois:

$$
\begin{gathered}
\frac{V_{1} V_{2} V_{3} V_{4} V_{5} V_{6} V_{7}}{C_{1} C_{2} C_{3} C_{4} C_{5} C_{6} C_{7}} M= \\
\frac{(36,22) \cdot(36,20) \cdot(36,20) \cdot(36,20) \cdot(36,10) \cdot(36,28) \cdot(36,00)}{(35,60) \cdot(36,30) \cdot(36,30) \cdot(36,25) \cdot(36,20) \cdot(36,10) \cdot(36,19)} M \\
\cong 1,007338 \cdot M
\end{gathered}
$$

A Figura 3 ilustra o comportamento das ações da empresa $\mathrm{C}$.

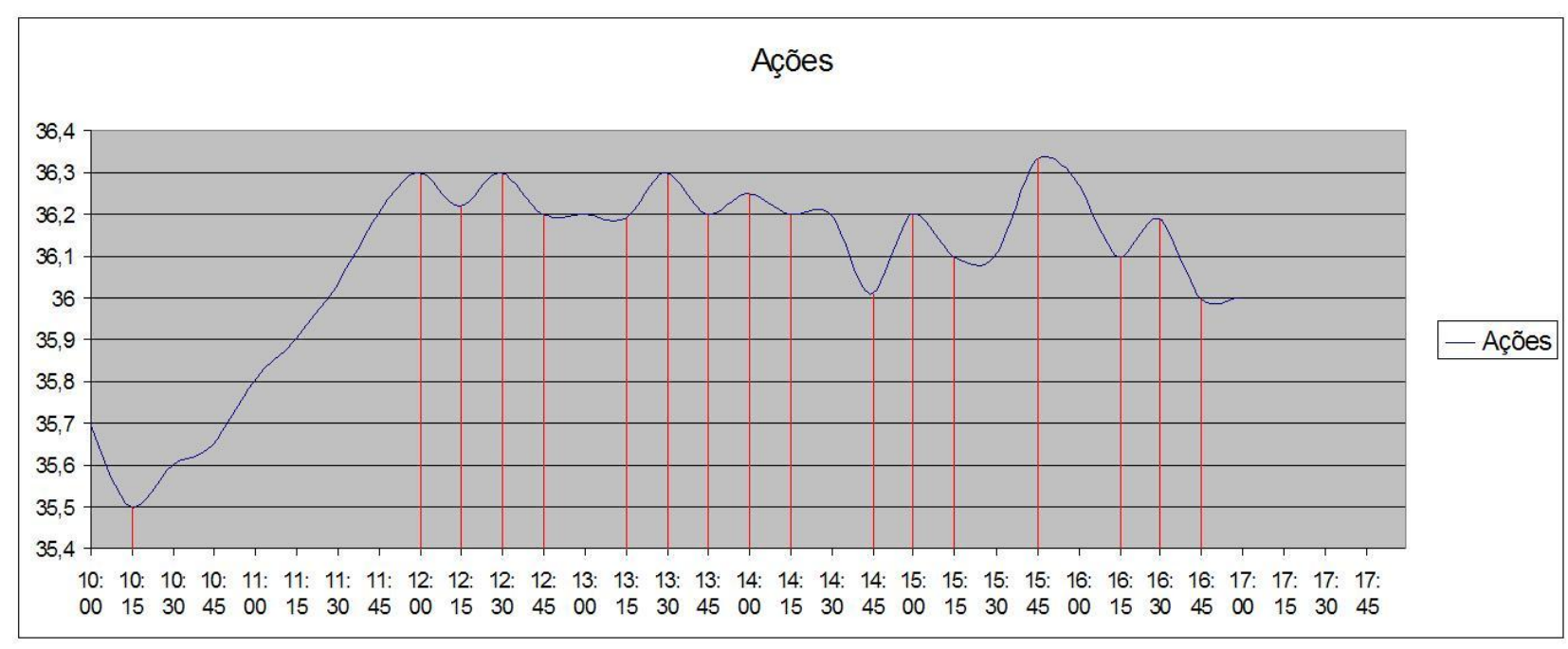

Figura 3. Indicações dos pontos críticos das oscilações nas ações da empresa C.

\section{Conclusões}

No decorrer do desenvolvimento deste trabalho, foram encontradas barreiras significativas para a aplicação de conceitos matemáticos muitas vezes abstratos em problemas de investimento em ações de empresas do agronegócio, nosso objeto de estudo.

Neste sentido, o maior obstáculo encontrado foi o de adaptar todos os resultados de funções contínuas nos quadros de ações das empresas consideradas, pois estes eram discretos. Assim, utilizando a teoria de sistemas discretos já existentes e desenvolvendo novos resultados, foi possível realizar o presente estudo e obtendo simulações altamente satisfatórias economicamente.

As análises e determinações dos momentos de compra e vendas foram realizadas a partir de teorema inéditos desenvolvidos neste trabalho. São eles: "Teste da Derivada Primeira Discreta para extremos relativos" e "Teste da Derivada Segunda Discreta para extremos relativos".

Nas simulações reais efetuadas, se 0 investidor procedesse transações comerciais de acordo com o método, seria obtido um lucro de 1,2\% em ações da empresa $A, 1,4 \%$ na empresa B e $0,7 \%$ na empresa $C$ nas datas escolhidas para a análise. Vale ressaltar que tais datas foram 
escolhidas por não apresentarem oscilações aparentemente significativas.

Assim, para a utilização do método, devem ser observadas também variáveis externas que impliquem grandes oscilações ou variações no valor das bolsas, ou seja, devem ser levadas em consideração outras variáveis que alterem o comportamento das ações.

Como o lucro obtido diário nas simulações foi próximo a $1 \%$, concluí-se que o método é extremamente eficiente, visto que este é o rendimento aproximado mensal de fundos em geral existentes no mercado financeiro.

Acredita-se que novos rumos de estudos em investimento em empresas do agronegócio na bolsa de valores a partir deste trabalho possam vir a ser de grande importância no meio acadêmico e operacional nas empresas e em investimento neste setor.

\section{Referências}

GABRIEL FILHO, L. R. A. Comportamento assintótico de sistemas lineares discretos. 2004. 65 p. Dissertação (Mestrado em Matemática) - Instituto de Ciências Matemáticas e Computação, Universidade de São Paulo. São Carlos.

LEITHOLD, L. O Cálculo com geometria analítica. 3.ed. v.1, São Paulo: Harbra, 1994. $684 \mathrm{p}$.

LEITHOLD, L. O Cálculo com geometria analítica. 3.ed. v.2, São Paulo: Harbra, 1994. $490 \mathrm{p}$.

MEDEIROS, P. T. O que é o mercado de ações. 3.ed. Rio de Janeiro: Simposium Consultoria e Serviços Técnicos, 1987. 